\title{
Evaluation of turbulent parameters based on angle-of-arrival fluctuation Yang $\mathrm{LI}^{1}$,Chao $\mathrm{GAO}^{2}$, Yi-Ming $\mathrm{LI}^{2}$, Gang $\mathrm{YANG}^{2}$ \& Xiao-Feng $\mathrm{LI}^{2}$ \\ ${ }^{1}$ Accounting School, Southwestern University of Finance and Economics, Chengdu, China \\ ${ }^{2}$ School of Astronautics and Aeronautic, University of Electronic Science and Technology of China, Chengdu, China
}

KEYWORD: laser propagation in the atmosphere, angle-of-arrival fluctuation, correlation model for evaluation, comprehensive performance of transmission characteristics

ABSTRACT: This paper investigates the feasibility of adopting single quantified parameter of atmospheric turbulence effect to evaluate others. To enrich and expand the test method on the transmission characteristics of atmospheric turbulence, this method could greatly reduce both the workload and costs of synthetic tests on the laser transmission characteristics, which has very positive and important significances for applications of space optical communication. The correlation models among the quantified parameters of atmospheric turbulence effects is deduced. The experimental results indicates that the angle-of-arrival fluctuation is related to other atmospheric turbulence effects based on the theory of Kolmogorov turbulence. Thus, other quantified parameters of atmospheric turbulence effects could be evaluated via acquired data of angle-of-arrival fluctuation.

\section{INTRODUCTION}

The characteristics of laser propagation through the turbulent atmosphere has been attracting increasing attentions in the field of quantitative analyses for suspended particles. Based on the large amount of statistical data collected from actual environment in long term, the mathematical models could be built to direct the development of instrumentations for atmospheric pollution testing (Andrews 2005, Rao 2005).

The tests for the characteristics of laser propagation usually last for 24 hours a day, and 7 days a week without intermission. For accuracy, the sample data for further analyses should be collected in similar conditions, and the number of samples should be greater than or equal to ten.

Currently, the China government has been building many localized testing sites for atmospheric pollution in large-scale. The test work in some testing sites has accumulated a large number of data.

The following work should be conducted for estimating the characteristics of laser propagation based on collected data and thus estimating the degree of atmospheric pollution.

1) Accomplish the correlation analyses for the testing data in the same area. Build the estimation models for the characteristics of laser propagation. The models should be validated and optimized.

2) Accomplish the correlation analyses for the testing data in different areas. Build the estimation models for the characteristics of laser propagation, which have more applicability.

Both the work are still in the initial stage.

The comprehensive characteristics of laser propagation include atmospheric attenuation, atmospheric turbulence, and meteorology. There are at least twelve important parameters in total. To test these parameters simultaneously will lead to huge workload and also make the analyses many and miscellaneous with trifles.

To reduce the workload of tests for the characteristics of laser propagation, an alternative approach is to use the statistics and the econometrics. Based on the relationships among the parameters for the characteristics of laser propagation, some certain parameters could be estimated by a few other parameters. Undoubtedly, this method could largely cut the costs for testing, and promote the development of instrumentations for atmospheric pollution testing.

\section{CORRELATION MODELSFOR CHARACTERISTICS OF LASER PROPAGATION}

The stochastic fluctuation of the atmospheric refractivity would lead to the variation of optical phase of laser propagation through the turbulent atmosphere. The common turbulence effects include angle-of-arrival fluctuation, irradiance scintillation, beam wander, and beam spreading (Toselli 2009, Gudimetla 2014). 
Angle-of-arrival fluctuations of an optical wave in the plane of the receive aperture are associated with image jitter in the focal plane of an imaging system. Fluctuations in received irradiance resulting from propagation through atmospheric turbulence is commonly described as "scintillation", which includes the temporal variation in received irradiance and spatial variation within a receiver aperture. In the presence of optical turbulence, a finite optical beam will experience random deflections as it propagates, causing further spreading of the beam by large-scale inhomogeneity of the atmosphere, and the instantaneous center of the beam is therefore randomly displaced in the receiver plane, producing what is commonly called beam wander. Beam spreading at the receiver plane can be modeled as if it arises from a random tilt angle at the transmitter plane, similar to angle-of-arrival fluctuations of a reciprocal propagating wave with the receiver diameter replaced by the transmitter beam diameter (Cui 2015).

According to the Kolmogorov spectrum, the theoretical models of the four turbulence effects for the plane wave are given below (Kolmogorov 1991).

1) Angle-of-arrival fluctuation:

$\sigma_{\alpha}^{2}=2.92 C_{n}^{2} D^{-1 / 3} L$

2) Irradiance scintillation:

$\sigma_{I}^{2}=1.23 C_{n}^{2} k^{7 / 6} L^{11 / 6}$

3) Beam wander:

$\sigma_{r}^{2}=0.97 C_{n}^{2} D^{-1 / 3} L^{3}$

4) Beam spreading:

$W^{2}=0.13 D^{2}+6.3\left(C_{n}^{2}\right)^{6 / 5} k^{2 / 5} L^{16 / 5}$

where $C_{n}^{2}$ is the atmospheric structure constant of refractive index, $D$ is the optical antenna aperture, $L$ is the propagation optical path length, $k=2 \pi / \lambda$ is the angular wave-number, $\lambda$ is the wavelength.

According to these theoretical models, angle-of-arrival fluctuation, irradiance scintillation, beam wander, and beam spreading are associated with each other turbulence effects by the atmospheric structure constant of refractive index. On the condition of fixed de-signed parameters for certain optical communication system, it is possible to estimate one turbulence effect by other turbulence effect.

Based on Equation 1-4, the correlation models between angle-of-arrival fluctuation and other turbulence effects are deduced below.

1) Irradiance scintillation:

$\sigma_{I}^{2}=0.42 D^{1 / 3} k^{7 / 6} L^{5 / 6} \sigma_{\alpha}^{2}$

2) Beam wander:

$\sigma_{r}^{2}=0.33 L^{2} \sigma_{\alpha}^{2}$

3) Beam spreading:

$W^{2}=0.13 D^{2}+1.74 D^{2 / 5} k^{2 / 5} L^{2}\left(\sigma_{\alpha}^{2}\right)^{6 / 5}$

\section{COLLECTED DATA OF THE CHARACTERISTICS OF LASER PROPAGATION}

The laboratory of space optical communication technology in University of Electronic Science and Technology of China (UESTC) has been conducting the testing of the characteristics of laser propagation since 2011. The testing channel locates near the Qingshuihe Campus of UESTC, which is in the suburb of Chengdu. The length of testing channel is $2.7 \mathrm{~km}$, and the wavelength is $830 \mathrm{~nm}$. The tests last for 24 hours a day, and 7 days a week without intermission. The collection and storage of generated data are full automatic.

This paper use the data collected from 6 a.m. to 8 a.m. on March, April, and May (92 days in total) of 2013 year. There are approximate 1800 samples for each turbulence effect, which satisfies the requirements for statistical analyses.

The weather of Chengdu between March and May is relative stability, and the channel between 6:00 and 8:00 is relatively good. On this note, those samples used in this paper have litter singular 
values, and the analytical results could be beneficial to the estimation of the characteristics of laser propagation in other areas.

Figure 1-4 depicts the collected data of angle-of-arrival fluctuation, irradiance scintillation, beam wander, and beam spreading from 6 a.m. to 8 a.m. on March, April, and May of 2013 year in Chengdu.

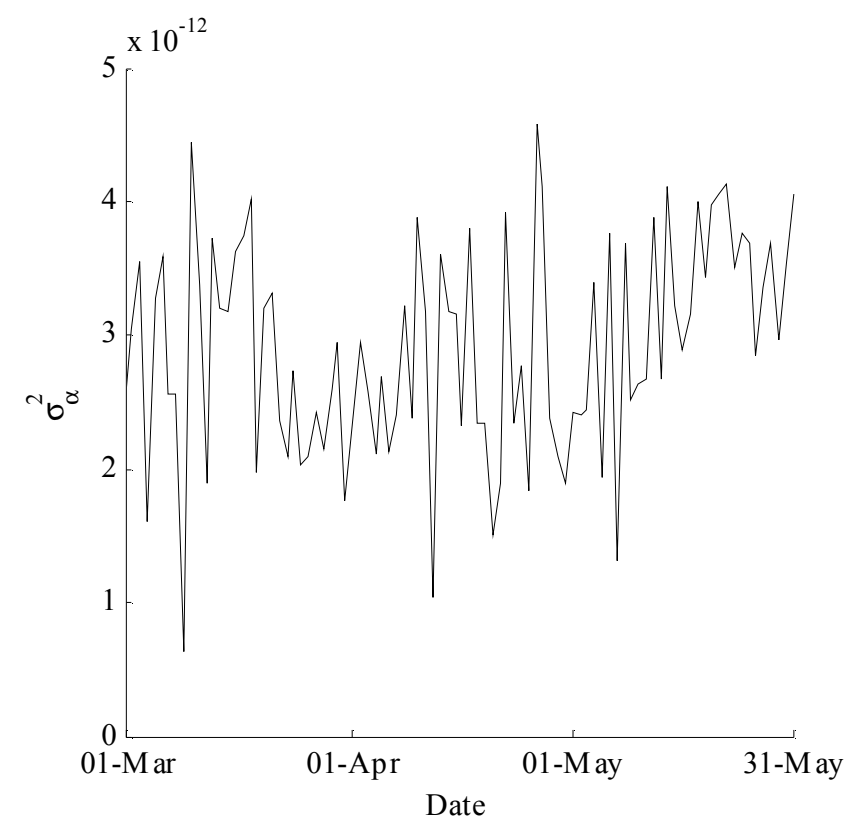

Figure 1.Collected data of angle-of-arrival fluctuation.

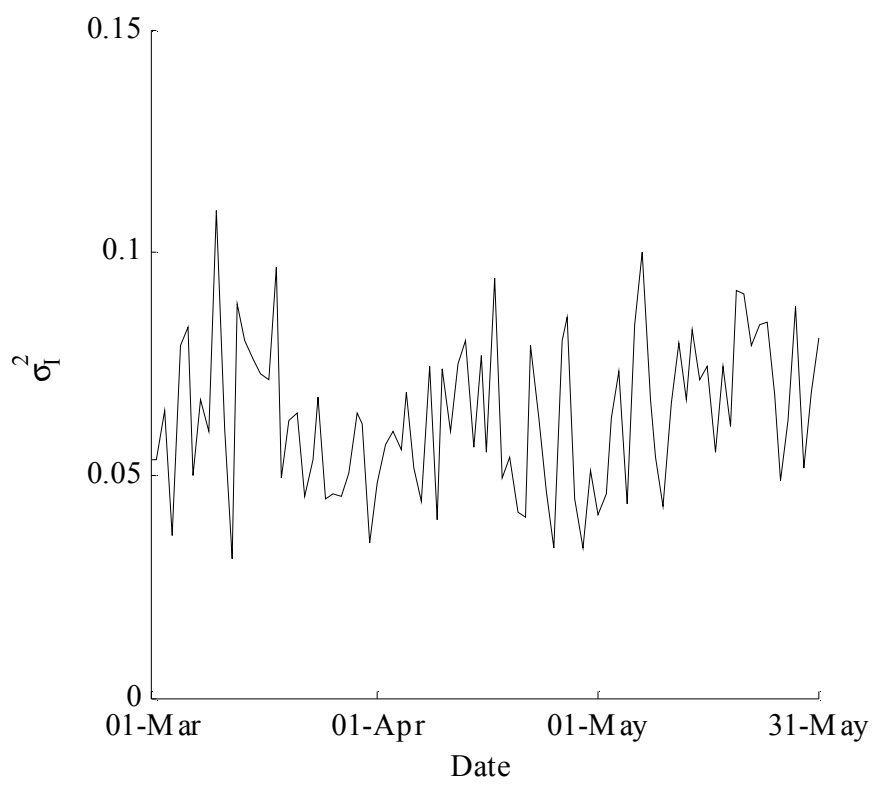

Figure 2. Collected data of irradiance scintillation. 


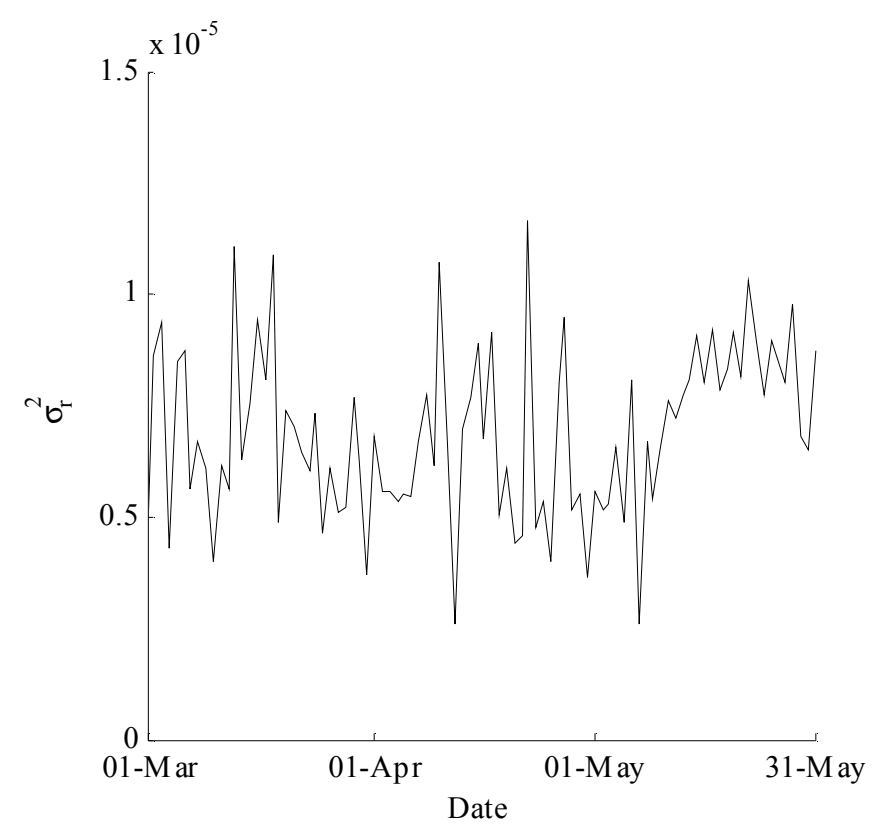

Figure 3. Collected data of beam wander.

\section{COMPARISON OF ESTIMATED VALUES AND COLLECTED DATA}

Based on the correlation models between the angle-of-arrival fluctuation and other turbulence effects deduced in Equation 5-7, the curves of irradiance scintillation, beam wander, and beam spreading are computed, followed by the comparison of estimated values and collected data as shown in Figure 5-7.

We use the actual optical parameters of testing equipment for the calculations of the correlation models. The length of testing channel $L$ is $2.7 \mathrm{~km}$, the wavelength $\lambda$ is $830 \mathrm{~nm}$, and the optical antenna aperture $D$ is $0.6 \mathrm{~m}$.

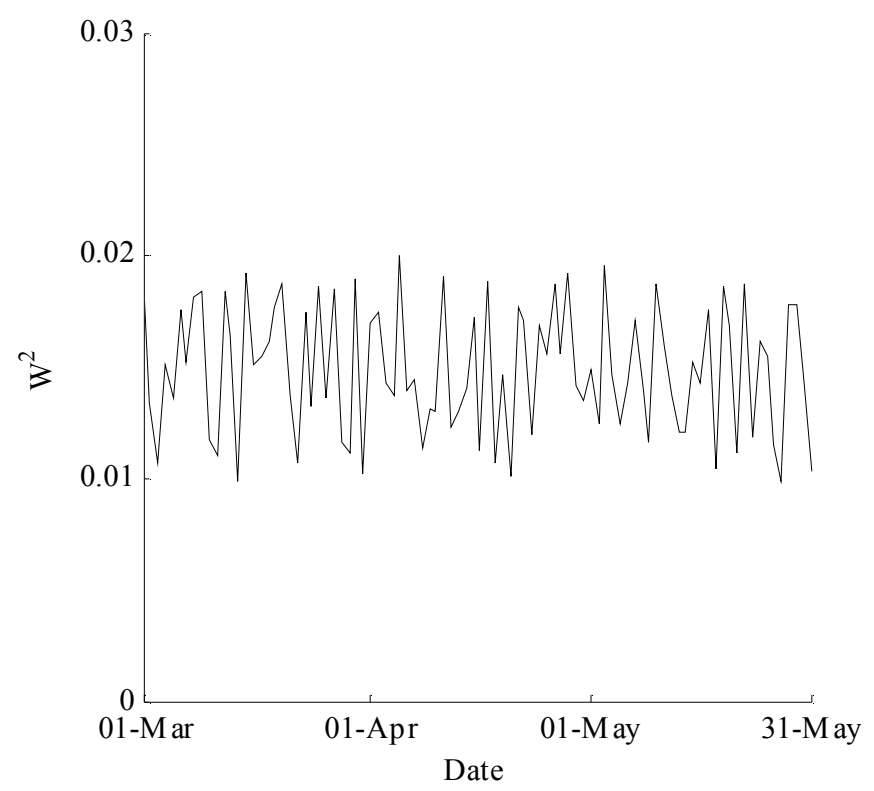

Figure 4. Collected data of beam spreading. 


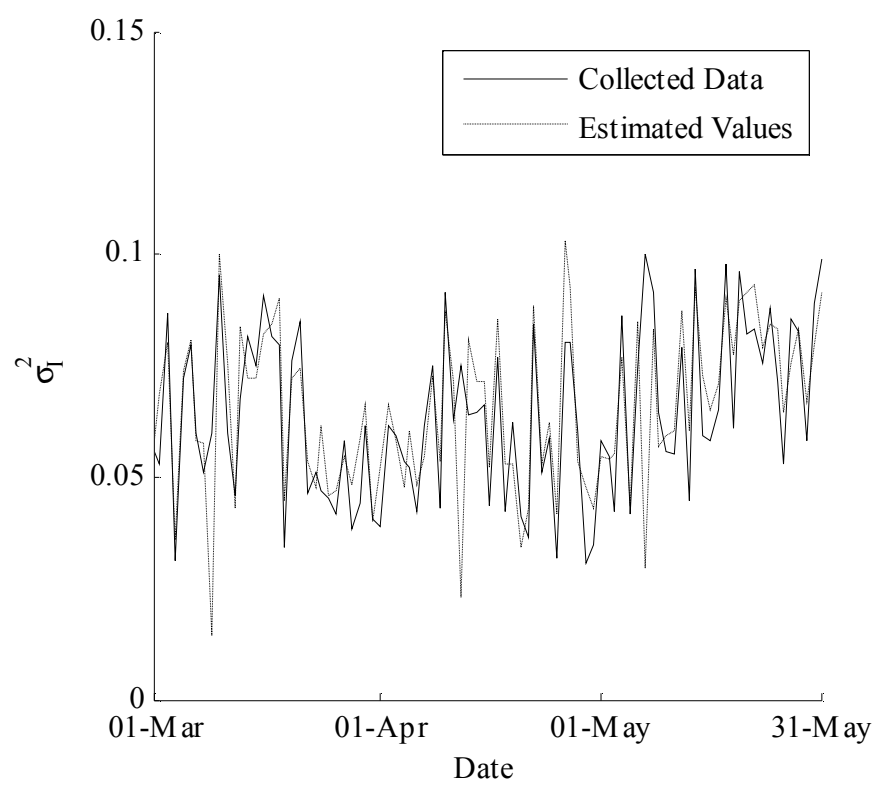

Figure 5. Comparison of estimated values and collected data of irradiance scintillation.

Table 1. Estimating Errors of Correlation Models based on Angle-of-Arrival Fluctuation

\begin{tabular}{ccc}
\hline & absolute errors & relative errors \\
\hline$\sigma_{I}^{2}$ & $8.546 \mathrm{e}-3$ & $19.13 \%$ \\
$\sigma_{r}^{2}$ & $9.719 \mathrm{e}-7$ & $16.02 \%$ \\
$W^{2}$ & $2.850 \mathrm{e}-3$ & $17.60 \%$ \\
\hline
\end{tabular}

The results indicate that the estimated values match collected data. Further investigations involve the errors about the correlation models, as shown in Table 1. The absolute and relative errors of irradiance scintillation are $8.546 \mathrm{e}-3$ and $19.13 \%$, respectively. The absolute and relative errors of beam wander are $9.719 \mathrm{e}-7$ and $16.02 \%$, respectively. The absolute and relative errors of beam spreading are $2.850 \mathrm{e}-3$ and $17.60 \%$, respectively.

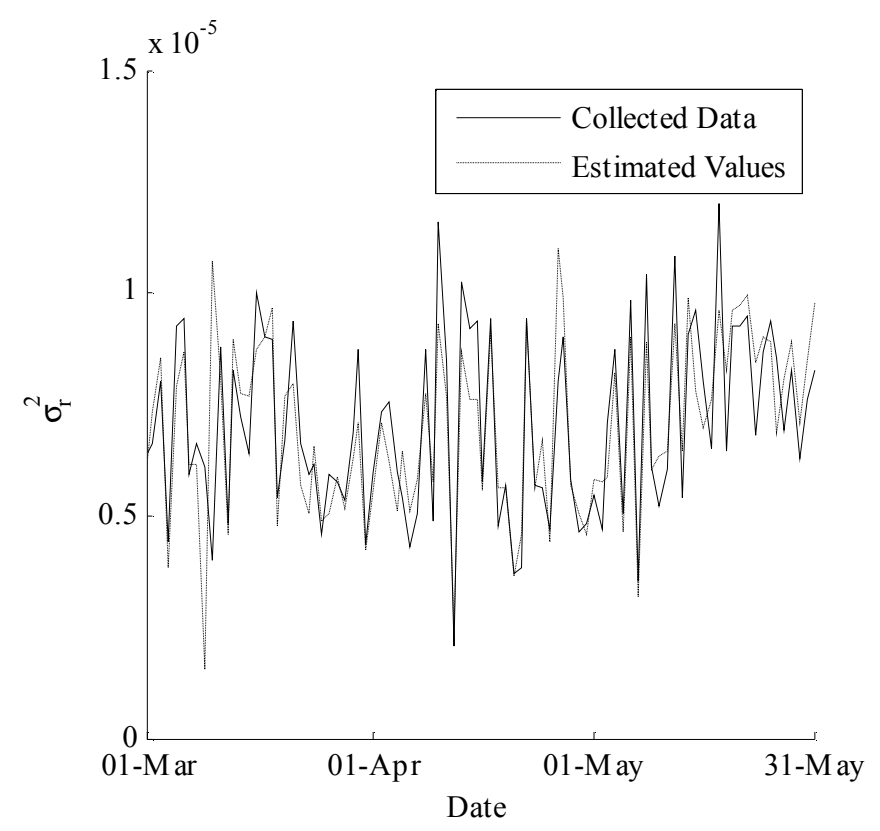

Figure 6. Comparison of estimated values and collected data of irradiance scintillation. 


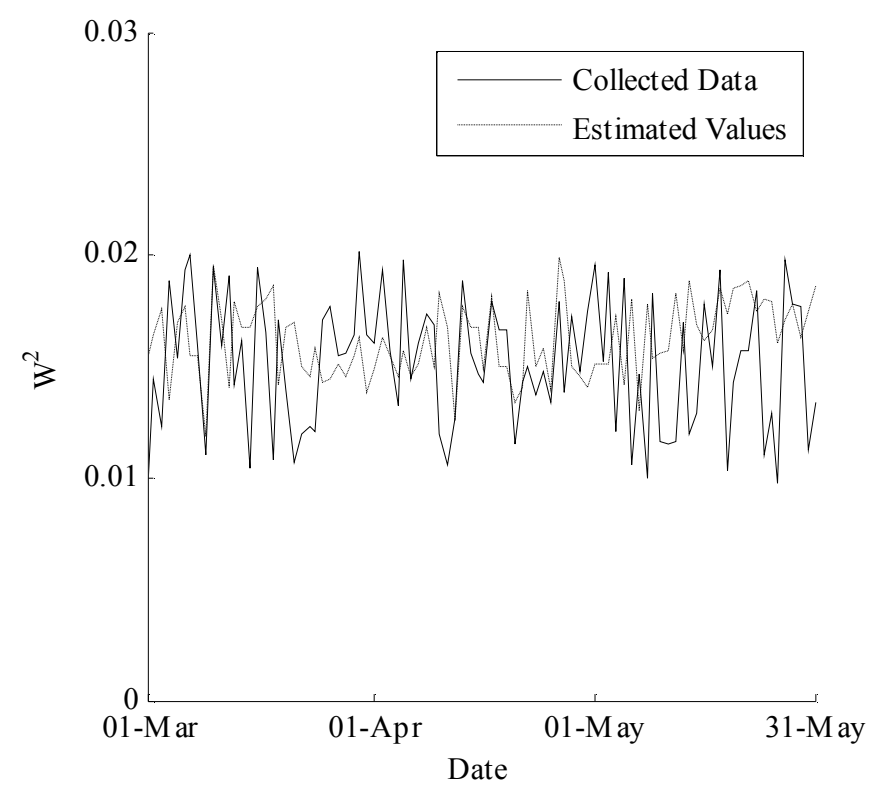

Figure 7. Comparison of estimated values and collected data of irradiance scintillation.

The stochastic fluctuation of atmospheric refractivity will lead to the stochastic fluctuation of wave front phase when propagation. Based on the Kolmogorov spectrum, there are certain relevancies between the angle-of-arrival fluctuation and other turbulence effects. It is available to estimate other turbulence effects for further analyses by collecting the data of angle-of-arrival fluctuation.

\section{CONCLUSION}

This paper adopts the collected data of angle-of-arrival fluctuation to evaluate other turbulence effects such as irradiance scintillation, beam wander, and beam spreading. This method could enrich and expand the testing method on the transmission characteristics of atmospheric turbulence.

Based on the correlation models and the actual optical parameters of testing equipment, the relative errors of estimated values for irradiance scintillation, beam wander, and beam spreading are smaller than $20 \%$.

It must be pointed that the collected data of angle-of-arrival fluctuation is relevant to the actual parameters of testing equipment, on which the quantitative analyses are based. Nevertheless, the method proposed in the paper is not limited to the actual parameters of testing equipment.

\section{REFERENCES}

[1] Andrews, L.C. 2005. Laser Beam Propagation through Random Media, 2nd. Bellingham: SPIE Press Monograph.

[2] Cui, L. 2015. Analysis of marine atmospheric turbulence effects on infrared imaging system by angle of arrival fluctuations. Infrared Physics and Technology 68(1): 28-34.

[3] Gudimetla, V.S.R, Holmes, R.B., \&Riker, J.F. 2014. Analytical expressions for the logamplitude correlation function for spherical wave propagation through anisotropic nonKolmogorov atmosphere. Journal of the Optical Society of America A: Optics, Image Science, and Vision 31(1): 148-154.

[4] Kolmogorov, A.N. 1991. The local structure of turbulence in incompressible viscous fluid for very large Reynolds numbers. Proceedings of the Royal Society A 434(1890): 9-13.

[5] Rao, R. 2005. Light Propagation in the Turbulent Atmosphere. Hefei: Anhui Science and Technology Press.

[6] Toselli. I., Andrews, L.C., Phillips, R.L., \&Ferrero, V. 2009. Free space optical system performance for a Gaussian beam propagating through non-Kolmogorov weak turbulence. IEEE Transactions on Antennas and Propagation 57(6): 1783-1788. 